\title{
Tumor microRNA-29a expression and the risk of recurrence in stage II colon cancer
}

\author{
ALINA WEISSMANN-BRENNER ${ }^{1,2^{*}}$, MICHAL KUSHNIR ${ }^{3 *}$, GILA LITHWICK YANAI $^{3}$, \\ RANIT AHARONOV $^{3}$, HADAS GIBORI $^{3}$, OFER PURIM ${ }^{1,2}$, YULIA KUNDEL $^{1,2}$, \\ SARA MORGENSTERN ${ }^{4,2}$, MARISSA HALPERIN $^{5,2}$, YARON NIV $^{6,2}$ and BARUCH BRENNER ${ }^{1,2}$ \\ ${ }^{1}$ Institute of Oncology, Davidoff Center, Rabin Medical Center, Beilinson Hospital, Petah Tikva; \\ ${ }^{2}$ Sackler Faculty of Medicine, Tel Aviv University, Tel Aviv; ${ }^{3}$ Rosetta Genomics Ltd., Rehovot; ${ }^{4}$ Institute of Pathology, \\ Rabin Medical Center, Beilinson Hospital; ${ }^{5}$ Institute of Pathology, Rabin Medical Center, Golda Hospital, \\ Petah Tikva; ${ }^{6}$ Institute of Gastroenterology, Rabin Medical Center, Beilinson Hospital, Petah Tikva, Israel
}

Received November 9, 2011; Accepted December 23, 2011

DOI: 10.3892/ijo.2012.1403

\begin{abstract}
There is emerging evidence for the prognostic role of various microRNA (miRNA) molecules in colon cancer. The aim of this study was therefore to compare the miRNA profiles in the primary tumor of patients with recurrent and non-recurrent colon cancer. The study population included 110 patients, $51(46 \%)$ with stage I and 59 (54\%) with stage II disease, who underwent curative colectomies between 1995 and 2005 without adjuvant therapy and for whom reliable miRNA expression data were available. RNA was extracted from formalin-fixed paraffin-embedded (FFPE) tumor samples. Initial profiling, using microarrays, was done in order to identify potential biomarkers of recurrence. The miRNA expression was later verified by quantitative real-time polymerase chain reaction (qRT-PCR). Findings were compared between patients who had a recurrence within 36 months of surgery (bad prognosis group, $n=23,21 \%$ ) and those who did not (good prognosis group, $\mathrm{n}=87,79 \%$ ) in the entire group and within each stage. The results showed that in stage I, none of the 903 miRNAs tested showed differential expression between patients with good prognosis compared with those with poor prognosis. In contrast, in stage II, one miRNA, miR-29a, showed a clear differential expression between the groups $(p=0.028)$. High expression of miR-29a was associated with a longer disease-free survival (DFS), on both univariate and multivariate analyses. Using miR-29a, the positive predictive value for non-recurrence was $94 \%$ (2 recurrences
\end{abstract}

Correspondence to: Dr B. Brenner, Institute of Oncology, Davidoff Center, Rabin Medical Center, Beilinson Campus, Petah Tikva 49100, Israel

E-mail: brennerb@clalit.org.il

Dr R. Aharonov, Rosetta Genomics Ltd., Rehovot 76706, Israel

E-mail: ranit_ah@rosettagenomics.com

${ }^{*}$ Contributed equally

Key words: microRNA, colon cancer, prognostic factors among 31 patients). The differential expression of miR-29a was verified by qRT-PCR, showing a similar impact of this miR on DFS. In conclusion, this study demonstrated a significant impact of miR-29a on the risk of recurrence in patients with stage II but not in patients with stage I colon cancer. Based on these results, a validation study is planned.

\section{Introduction}

Colorectal cancer (CRC) is one of the most common malignancies; globally, 608,000 patients with CRC die annually, making $\mathrm{CRC}$ the third most common cause of cancer-related death worldwide (1). Surgical resection is the standard treatment of localized CRC but the risk of recurrence following successful complete (R0) resection is high in stage II and III disease and most of these patients will therefore receive additional treatment: postoperative (adjuvant) chemotherapy if their tumor was located in the colon and preoperative (neoadjuvant) or postoperative (adjuvant) chemoradiotherapy if their tumor was located in the rectum. However, the majority of patients receiving neo/adjuvant therapy, which is associated with significant morbidity and even long-term sequelae and mortality, do not need it.

Current methods for stratifying CRC patients by their prognosis and thereby identifying those who need neo/adjuvant therapy are still mainly based on the extent of the local tumor spread, i.e. the TNM staging (2). Other clinicopathological prognostic factors, such as tumor grade, vascular invasion and perineural spread as well as the presence of obstruction or perforation, add little to our capacity to distinguish between patients who do and those who do not need additional treatment (3). Multiple efforts have been made to utilize biological markers in order to improve our ability to determine the prognosis of an individual patient and thereby to improve the selection of treatment. Recently, the use of microsatellite instability (MSI) and gene expression profile (Oncotype Dx colon) have shown a potential in this field but their actual impact on treatment assortment is still unclear (4). There is a clear need therefore for novel prognostic factors in CRC using newer technologies. 
microRNAs (miRNAs) are short non-coding RNAs, 17-22 nucleotides in length, that regulate gene expression and thereby play significant roles in human development and various physiological and pathological processes (5-7). The expression of miRNAs is dynamic and corresponds to changes in the physiological situation. This feature raises the possibility that profiles of miRNAs derived from tumoral specimens may be used as diagnostic or prognostic biomarkers (8-11). Recent studies, also from our group, indeed showed the association between the expression of various miRNAs and patient outcome in several hematological and solid malignancies, including in the gastrointestinal tract (12-18). Currently, the prognostic role of miRNAs in CRC is unclear but emerging data are encouraging (18-21).

The aim of this study was to compare the miRNA profiles in the initial surgically resected specimens of patients with recurrent and non-recurrent CRC and thereby to evaluate the prognostic impact of various miRNAs. For uniformity purposes and because rectal cancer patients are usually treated even before surgery, this study focused on tumors located in the colon itself.

\section{Patients and methods}

Patients. The study population consisted of patients with histologically confirmed adenocarcinoma of the colon that were operated and followed at the two hospitals of the Rabin Medical Center (RMC), Beilinson and Golda, in Petach Tikva, Israel. Patients with tumors in the recto-sigma region were eligible but those with rectal tumors were excluded. All patients had stage I-II disease according to the AJCC staging system version 6 (2) and underwent curative resections with clear margins (R0 resection). Eligible patients had been operated during a 10-year period, from 1995 until 2005 , to ensure the quality of the surgical specimens, adequate follow-up and relatively uniform methods for detection of recurrence in all patients. Patients who received any postoperative adjuvant therapy were excluded, to allow evaluation of a pure prognostic effect rather than a predictive effect of the relevant miRNAs. For the same reason, patients with stage III disease, who usually receive adjuvant therapy, were also excluded. To assess the patients' 3-year disease-free survival (DFS), a minimum follow-up of three years for nonrecurring patients was required. Eligible patients, fulfilling all these criteria, were identified from the databases of the Institutes of Oncology at both hospitals, following the study's approval by the institutional ethics committee.

Follow-up. As a retrospective study, the follow-up policy was not pre-specified in the protocol; still, patients were followed every three to six months in the first three years, regardless of the stage of their disease, according to hospital policy. Patients were then followed every six months for two years and yearly afterwards. Follow-up visits consisted of a medical history, physical examination and testing of the carcinoembryonic antigen (CEA) serum level. During the first three years patients with stage II disease had also abdominal ultrasound and chest $\mathrm{X}$-rays or chest, abdominal and pelvic computerized tomography (CT) scan once yearly. Colonoscopy was done one year from surgery and every 3-5 years afterwards. The study group was divided into those with 'recurrent' and those with 'non- recurrent' experience of recurrence of disease within the first three years of follow-up. Time of events (recurrence or death) is defined from the date of surgery.

Pathology. Paraffin-embedded blocks of the surgical specimens from the initial colectomies of the eligible patients were retrieved from the archives of the Institutes of Pathology at both hospitals of the RMC. For the purpose of this study, only blocks containing at least $50 \%$ tumor and $>0.5 \mathrm{~cm}$ in diameter were considered suitable for evaluation. From each block, 10 slices of $10 \mu \mathrm{m}$ each were collected in one $1.5 \mathrm{ml}$ tube for RNA extraction and microRNA analysis. In addition, a $4 \mu \mathrm{m}$ slide dyed with hematoxylin and eosin (H\&E) was prepared from each block. All H\&E slides were reviewed by two expert pathologists (SM, MH), who confirmed the diagnosis and the appropriateness for the study as well as other histological parameters, such as mucin secretion, grade and vascular or perineural invasion.

RNA extraction. Total RNA was extracted as described before (11). Briefly, the sample was incubated in xylene at $57^{\circ} \mathrm{C}$ to remove excess paraffin, and then was washed several times with ethanol. Protein degradation was performed by incubation of the sample in a proteinase $\mathrm{K}$ solution at $45^{\circ} \mathrm{C}$ for a few hours. The RNA was extracted using acid phenol/chloroform and then precipitated using ethanol; DNAses were introduced to digest DNA. Total RNA quantity and quality was measured by Nanodrop ND-1000 (NanoDrop Technologies, Wilmington, DE).

miRNA microarray platform. Custom miRNA microarrays were described before (11). Briefly, 900 DNA oligonucleotide probes representing miRNAs (Sanger database version 10 and additional miRNAs predicted and validated by Rosetta Genomics) were spotted in triplicate on coated microarray slides (Nexterion ${ }^{\circledR}$ Slide E, Schott, Mainz, Germany). Two types of positive controls were included in the experimental design: i) synthetic small RNAs were spiked into each RNA sample before labeling to verify labeling efficiency and ii) probes for abundant small RNAs were spotted to validate RNA quality.

Microarray signal calculation and normalization. The RNA fluorescence data from the slide corresponding to each patient was loaded into a single database. Two samples for which the expression levels were very low $(<10$ miRNAs with signals $>300$ ) were excluded. Microarray spots were combined and signals were normalized as described before (11). Data were log-transformed and subsequent analysis was performed in logspace. Henceforth, the expression level or signal of an individual miRNA refers to the $\log 2$ normalized value.

$q R T-P C R$. For a subset of 15 samples, hsa-miR-29a expression was quantified along with 4 miRNAs chosen from the microarray data as normalizers (hsa-miR-214, hsa-miR-221, hsa-miR-141, hsa-miR-185), using a quantitative real-time PCR method previously described (22). Samples were normalized by subtracting from the cycle threshold $(\mathrm{Ct})$ of hsa-miR-29a the average $\mathrm{Ct}$ of the normalizers for the sample, and adding back a scaling constant (the average $\mathrm{Ct}$ of the normalizers over the entire sample set). The $\mathrm{Ct}$ scale was then inverted such that 
high values represent high expression (as microarray fluorescence) by subtracting the resulting value from 50 .

Data analysis and statistics. The clinical and pathological data of eligible patients whose surgical specimens were deemed suitable for the tissue analysis were entered into an electronic database created for this purpose and anonymized. Individuals involved in the miRNA measurement were blinded to the patients' clinical data.

The data were split by prognosis grouping into patients that experienced or did not experience recurrence of disease within 36 months from surgery. A total of 147 miRNAs had a median signal that passed the minimal threshold of 300 in at least one group: 147 passed this threshold when restricting to stage I samples and 153 passed it when restricting to stage II samples. For each of these, the distributions of readings in the two groups were compared using the Student's t-test. The threshold for $\mathrm{p}$-value significance was selected by setting a Benjamini-Hochberg false-discovery rate (FDR) (23) of 0.1 . The fold-change between the two groups, which is the ratio of the median expression levels, was calculated for each miRNA and miRNAs were deemed differentially expressed if the p-value was below the significance threshold.

For the most significant miRNA, the cohort was split into three groups according to the expression signal of this miRNA. Kaplan Meier (KM) survival curves were then used to compare the groups, and p-values were obtained by comparing the upper and lower expression tertiles using the log-rank test. KM analysis was used to test the prognostic value of clinical and pathological factors and these, as well as miRNA readings, were combined using multivariate Cox regression to evaluate the independent prognostic significance of all factors. p-value of 0.05 was used as threshold for inclusion/exclusion of factors.

The overall goal of this research was to predict reliably non-recurrence after surgery, i.e. to achieve a high positive predictive value (PPV, number of correctly predicted nonrecurrent patients/all patients predicted to not recur). After choosing the most relevant miRNA, its predictive value was optimized by finding the threshold that maximized PPV with high sensitivity for detection of non-recurrence. KM was repeated based on this separation, and log-rank was repeated to measure separation.

\section{Results}

Patient characteristics. The clinicopathological characteristics of the study population are summarized in Table I. A total of 112 eligible patients were identified. Reliable miRNA expression data were obtained from the samples of 110 of these patients (98\%) and these patients were included in the statistical analysis. The median age of the entire study cohort was 76 years (range: 49-90) and 58\% were males. Fifty-one patients (46\%) had stage I disease at diagnosis, and 59 patients (54\%) had stage II. With a median follow-up of 78 months (range: 34-200 months) for non-recurring patients, a total of 23 patients $(21 \%)$ experienced recurrence of disease within three years from the date of surgery (poor prognosis group), and 87 (79\%) did not (good prognosis group). The 'good prognosis' group included 81 patients who did not have any recurrence during their follow-up and 6 who experienced a recurrence after more than 36 months from surgery.
Patients with stage I disease had a trend for a higher 3-year recurrence rate $(27 \%$ vs. $14 \%$ ) although this difference is not statistically significant $(\mathrm{p}=0.067)$. Considering the entire study population, none of the clinicopathological characteristics tested showed a significant difference in distribution between the good and poor prognosis groups. However, patients with stage II disease had a significantly higher rate of left-sided tumors in the 'poor prognosis' group ( $80 \%$ vs. $46 \%$, $\mathrm{p}=0.034$ ).

Association of miRNAs with outcome. Due to the differential prognosis for patients with different stages, we split our analysis between patients with stage II and those with stage I. As few of the latter experienced recurrence we a priori expected little prognostic power for miRNA. Indeed, none of the 903 miRNAs tested showed differential expression, following FDR correction, between patients with good prognosis compared with those with bad prognosis in stage I patients. In contrast, in stage II, one miRNA, miR-29a, came out with a clear differential expression (Fig. 1, p=6.4e-5) between patients with good prognosis $(n=43)$ and those with poor prognosis $(n=16)$, well surpassing the FDR-adjusted threshold $(\mathrm{p}=0.028)$. As a result, only stage II patients were analyzed here. Within this group, most recurrence-free patients $(67 \%)$ had normalized miR-29a expression value of 13.5 or higher while the same level of miR-29a expression was observed in only $12 \%$ of the patients with recurrent tumors (Fig. 1).

The prognostic value of the miR-29a signature in stage II patients was also evaluated in the context of commonly used clinico-pathological variables including tumor grade and patient age. By univariate analyses, miR-29a signature, tumor grade and location were found to be associated with prognosis (Table II). However, by a multivariate analysis, only the miR-29a signature retained its prognostic impact $(\mathrm{p}=1.6 \mathrm{e}-4)$, indicating that it was a strong prognostic factor (Table II).

We next compared the prognosis of patients in groups stratified according to miR-29a expression levels. We divided the samples into tertiles according to high, intermediate or low expression level and plotted the DFS curves for the three groups. Decreased level of miR-29a was associated with greater risk of recurrence (Fig. 2A). miR-29a expression was also found to differentiate a sub-group of these patients with very privileged prognosis and a sub-group with very poor prognosis (Fig. 2B).Using a lower threshold of miR-29a signal of 13.5 , allows the identification of a group $(n=31)$ with only two cases of recurrence within 3 years. The sensitivity for identifying non-recurrence was 67\% (95\% CI: 51-81\%) and the specificity was $88 \%$ (95\% CI: 62-98\%). The PPV for nonrecurrence was $94 \%$ (95\% CI: 79-99\%) and the NPV was $50 \%$ (95\% CI: 31-69\%). Similarly, an upper threshold of 13 can be assigned for miR-29a signals in order to identify a group $(n=6)$ with a very high rate of recurrence (5 cases) within 3 years. The sensitivity in this case for identifying recurrence was $31 \%$ (95\% CI: $11-59 \%$ ), the specificity was $98 \%$ (95\% CI: $88-100 \%)$, the PPV was $83 \%$ (95\% CI: $36-100 \%)$ and the NPV was $79 \%$ (95\% CI: 66-89\%). When dividing the data according to these thresholds, the resulting p-value is, as expected, very significant $(\mathrm{p}=1.5 \mathrm{e}-5)$.

PCR validation of miR-29a expression. In order to assess the validity of the detected miR-29a expression signature, we 
Table I. Clinicopathological characteristics.

\begin{tabular}{|c|c|c|c|c|c|c|c|c|c|c|}
\hline & \multicolumn{4}{|c|}{ All patients $(n=110)$} & \multicolumn{3}{|c|}{ Stage I $(n=51)$} & \multicolumn{3}{|c|}{ Stage II $(n=59)$} \\
\hline & $\begin{array}{c}\text { All }^{\mathrm{a}} \\
\text { patients } \\
(\mathrm{n}=110)\end{array}$ & $\begin{array}{c}\text { Good }^{\mathrm{b}} \\
\text { prognosis } \\
(\mathrm{n}=87)\end{array}$ & $\begin{array}{l}\text { Poor }^{\mathrm{b}} \\
\text { prognosis } \\
(\mathrm{n}=23)\end{array}$ & p-value ${ }^{c}$ & $\begin{array}{c}\text { Good }^{\mathrm{b}} \\
\text { prognosis } \\
(\mathrm{n}=44)\end{array}$ & $\begin{array}{c}\text { Poor }^{\mathrm{b}} \\
\text { prognosis } \\
(\mathrm{n}=7)\end{array}$ & p-value & $\begin{array}{c}\text { Good }^{\mathrm{b}} \\
\text { prognosis } \\
(\mathrm{n}=43)\end{array}$ & $\begin{array}{l}\text { Poor }^{\mathrm{b}} \\
\text { prognosis } \\
(\mathrm{n}=16)\end{array}$ & p-value \\
\hline \multicolumn{11}{|l|}{ Age, years } \\
\hline Median (range) & $76(49-90)$ & $76(49-90)$ & $76(57-90)$ & 0.921 & $73(49-89)$ & $76(69-80)$ & 0.233 & $79(53-90)$ & $77(57-90)$ & 0.133 \\
\hline \multicolumn{11}{|l|}{ Gender } \\
\hline Male & $64(58 \%)$ & $52(60 \%)$ & $12(52 \%)$ & 0.635 & $25(57 \%)$ & $5(71 \%)$ & 0.688 & $27(63 \%)$ & $7(44 \%)$ & 0.241 \\
\hline Female & $46(42 \%)$ & $35(40 \%)$ & $11(48 \%)$ & & $19(43 \%)$ & $2(29 \%)$ & & $16(37 \%)$ & $9(56 \%)$ & \\
\hline \multicolumn{11}{|l|}{ Location $^{\mathrm{a}, \mathrm{d}}$} \\
\hline Right colon & $48(45 \%)$ & $42(49 \%)$ & $6(29 \%)$ & 0.088 & $20(45 \%)$ & $3(50 \%)$ & 1 & $22(54 \%)$ & $3(20 \%)$ & 0.034 \\
\hline Left colon & $56(53 \%)$ & $41(49 \%)$ & $15(71 \%)$ & & $22(50 \%)$ & $3(50 \%)$ & & $19(46 \%)$ & $12(80 \%)$ & \\
\hline Unknown & $2(2 \%)$ & $2(2 \%)$ & $0(0 \%)$ & & $2(5 \%)$ & $0(0 \%)$ & $0(0 \%)$ & $0(0 \%)$ & & \\
\hline \multicolumn{11}{|l|}{ Grade $^{\mathrm{d}}$} \\
\hline I & $30(27 \%)$ & $22(25 \%)$ & $8(35 \%)$ & 0.431 & $17(39 \%)$ & $3(43 \%)$ & 1 & $5(12 \%)$ & $5(31 \%)$ & 0.116 \\
\hline II & $75(68 \%)$ & $61(70 \%)$ & $14(61 \%)$ & & $26(59 \%)$ & $4(57 \%)$ & & $35(81 \%)$ & $10(63 \%)$ & \\
\hline III & $5(5 \%)$ & $4(5 \%)$ & $1(4 \%)$ & & $1(2 \%)$ & $0(0 \%)$ & & $3(7 \%)$ & $1(6 \%)$ & \\
\hline \multicolumn{11}{|l|}{ Examined $\mathrm{LN}^{\mathrm{a}}$} \\
\hline$\geq 12$ & $33(31 \%)$ & $24(29 \%)$ & $9(39 \%)$ & 0.445 & $9(21 \%)$ & $0(0 \%)$ & 0.463 & $15(37 \%)$ & $9(56 \%)$ & 0.236 \\
\hline$<12$ & $74(69 \%)$ & $60(71 \%)$ & $14(61 \%)$ & & $34(79 \%)$ & $7(100 \%)$ & & $26(63 \%)$ & $7(44 \%)$ & \\
\hline Mucin secretion & $14(13 \%)$ & $11(13 \%)$ & $3(14 \%)$ & 1 & $4(9 \%)$ & $1(14 \%)$ & 0.546 & $7(17 \%)$ & $2(13 \%)$ & 1 \\
\hline \multicolumn{11}{|l|}{ Site of recurrence } \\
\hline Locoregional & $5(17 \%)$ & $1(17 \%)$ & $4(17 \%)$ & 1 & $1(25 \%)$ & $0(0 \%)$ & 0.236 & $0(0 \%)$ & $4(25 \%)$ & 1 \\
\hline Distant & $20(69 \%)$ & $3(50 \%)$ & $17(74 \%)$ & & $1(25 \%)$ & $7(100 \%)$ & & $2(100 \%)$ & $10(63 \%)$ & \\
\hline Combined & $4(14 \%)$ & $2(33 \%)$ & $2(9 \%)$ & & $2(50 \%)$ & $0(0 \%)$ & & $0(0 \%)$ & $2(12 \%)$ & \\
\hline
\end{tabular}

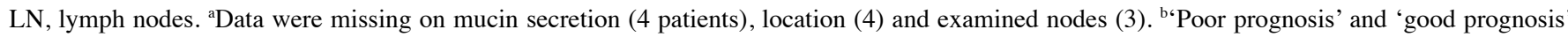
groups were defined as patients who either did or did not experience disease recurrence within 36 months of surgery. Patients whose disease recurred after more than 36 months from surgery were classified as having 'good prognosis'. 'P-value was computed for the 'poor prognosis' vs. the 'good prognosis' groups (using Mann-Whitney for continuous data and Fisher's exact test for contingency tables). ${ }^{\mathrm{d}} \mathrm{P}$-values were computed for right colon vs. left colon for 'Location', between grade I and grade II-III combined for 'Grade' and between Locoregional and Distant for 'Site of recurrence'.

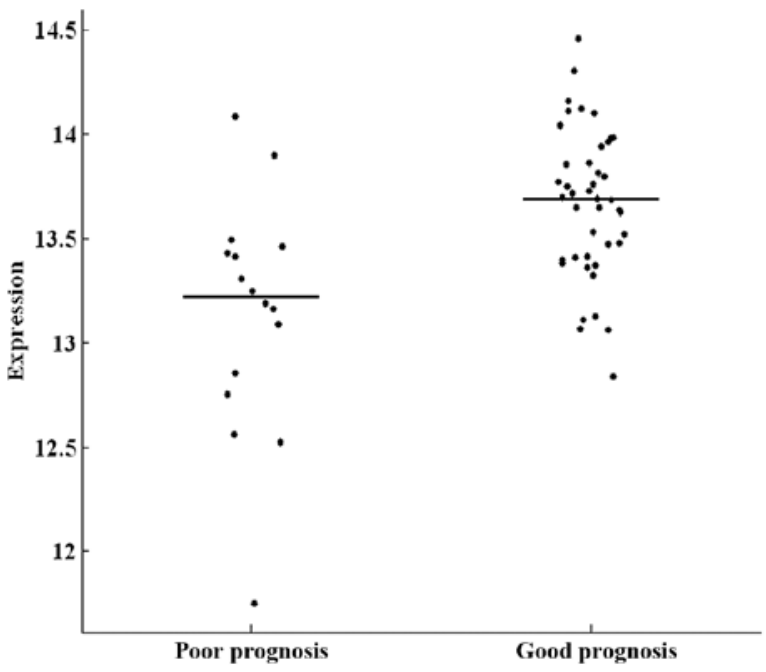

Figure 1. hsa-miR-29a expression in stage II good and poor prognosis patients. Dotplot representation of hsa-miR-29a expression [in normalized $\log 2$ (florescence units)] for patients with good prognosis (no recurrence within 36 months of surgery, $n=43$, median=13.7) and those with poor prognosis (recurrence within 36 months of surgery, $n=16$, median=13.2). The p-value for this comparison is $6.5 \mathrm{e}-5$ (t-test) and the fold change is 1.4 (between median values, shown as central lines). performed qRT-PCR for 15 samples that had been included in the microarray analysis that were differentially expressed. The relative expression level of miR-29a was correlated with the corresponding microarray signal intensities. The correlation coefficient between microarray expression and the PCR was fair (0.68) and miR-29a was significantly lower in the good prognosis group for both the custom array and the PCR (Fig. 3).

qRT-PCR data were analyzed also for prediction of DFS by KM analysis. As shown in Fig. 4, KM analysis using expression profiles demonstrated that the high and low miR-29a expression groups were significantly different in their DFS as measured by both microarray $(\mathrm{P}=0.004)$ and $\mathrm{qRT}-\mathrm{PCR}(\mathrm{P}=0.0039)$.

\section{Discussion}

The current study suggests a potential prognostic role of miR-29a in patients with resected stage II colon cancer. Patients who did not experience a recurrence within three years from the resection of their primary tumor had significantly higher expression levels of this miRNA compared with patients who did have a recurrence within the same time. To further support the prognostic role of miR-29a, high expression levels of this 
A

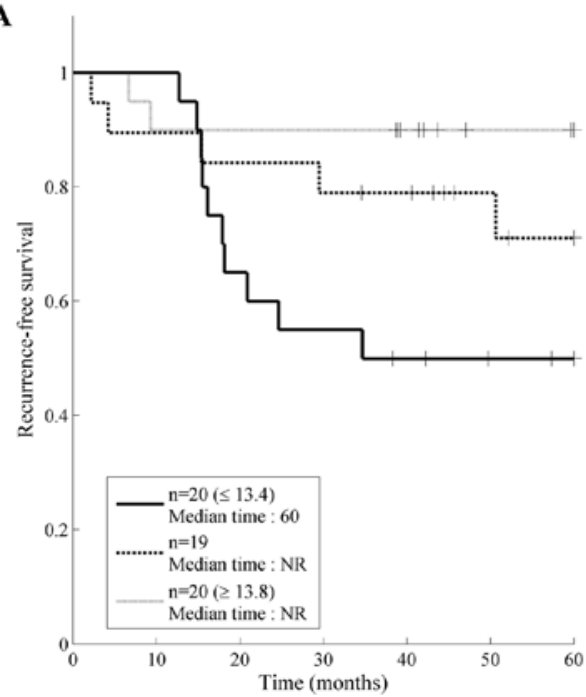

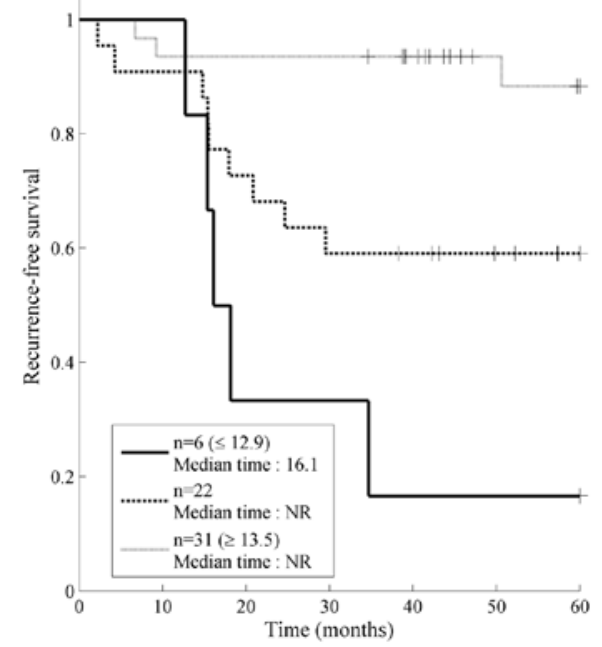

Figure 2. Kaplan-Meier model of recurrence for colon cancer patients using hsa-miR-29a expression. Population was split by hsa-miR-29a expression [in normalized $\log 2$ (florescence units)], based on expression tertiles (A) and based on best separation (B). P-values, as calculated between the two extreme expression tertiles using log-rank are 0.011 (A) and 1.5e-5 (B). NR, not reached. Gray crosses indicate censored patients.
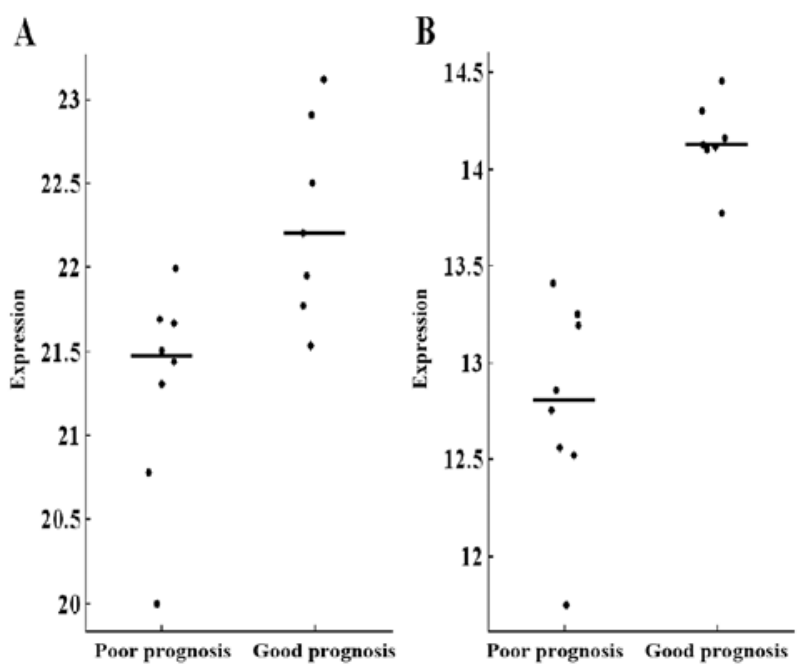

Figure 3. hsa-miR-29a expression for a subset of good and poor prognosis patients in PCR and microarray platforms. Dotplot representation of hsamiR-29a expression (inverted normalized Cts) is shown for a subset of 15 patients that were measured using PCR (A). The expression of these samples as measured in microarrays [normalized $\log 2$ (florescence units)] is shown as well (B). Central lines represent the median values.

miRNA correlated not only with the risk of recurrence but also with the duration of DFS. Finally, miR-29a preserved its powerful prognostic role also in a multivariate model encompassing common prognostic factors in colon cancer and in a validation test using qRT-PCR. All these findings were limited to stage II, and not relevant to stage I patients.

In recent years we have witnessed multiple reports on dysregulated expression of multiple miRNAs in CRC. Increased or decreased levels of various miRNAs were observed in the context of different clinical settings, including the diagnosis of the disease $(24,25)$, the evaluation of prognosis $(18-21)$ and the prediction of response to treatmenT $(26,27)$. Despite the flood of information, the data are still premature to establish the exact role
Table II. Risk of recurrence in stage II patients.

\begin{tabular}{|c|c|c|c|}
\hline \multirow[b]{2}{*}{ Variable } & $\log$ rank $^{a}$ & \multicolumn{2}{|c|}{ Multivariate $\operatorname{Cox}^{\mathrm{a}}$} \\
\hline & p-value & $\begin{array}{l}\text { Hazard ratio } \\
\quad(95 \% \mathrm{CI})\end{array}$ & p-value \\
\hline \multicolumn{4}{|l|}{ hsa-miR-29ab } \\
\hline ( $\log 2$ normalized expression) & 0.021 & $0.194(0.063-0.597)$ & $4.3 \mathrm{E}-03$ \\
\hline $\mathrm{Age}^{\mathrm{b}}$ & 0.5 & NA & NA \\
\hline Gender & 0.124 & NA & NA \\
\hline Grade $^{c}$ & 0.03 & $0.795(0.208-3.04)$ & 0.737 \\
\hline Tumor location $^{\mathrm{c}}$ & 0.043 & $2.74(0.744-10.1)$ & 0.13 \\
\hline Examined lymph nodes ${ }^{c}$ & 0.163 & NA & NA \\
\hline Mucin secretion & 0.913 & NA & NA \\
\hline
\end{tabular}

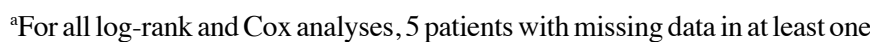
variable were not included. Only variables with $\log$-rank $\mathrm{P}<0.05$ were taken for the multivariate Cox analysis. For variables not included, the Hazard ratio and p-value are marked NA (not applicable). ${ }^{b}$ Log-rank p-values for hsa-miR-29a expression and for age were obtained by running log-rank on the two most extreme tertiles. 'P-values were computed between grade I and grade II-III combined for 'Grade', right colon vs. left colon for 'Tumor location', $\geq 12$ lymph nodes examined vs. $<12$ lymph nodes examined for 'Examined lymph nodes'.

of any miRNA in CRC. The focus of the current study was the prognostic role of miRNAs in colon cancer. To date, the miRNA most studied in this setting is miR-21. Upregulation of miR-21 was shown to correlate with lymph node positivity, advanced stage, development of distant metastases and shorter survival $(21,28,29)$. Other miRNAs were also reported to correlate with tumor stage or patient outcome, or both, including for instance miR-18a, miR-31, miR-106a, miR-200c, miR-143, miR-145, miR-320 and miR-498 (19-21,28-31). Our study failed to confirm the prognostic role of any of these miRNAs while demon- 
A

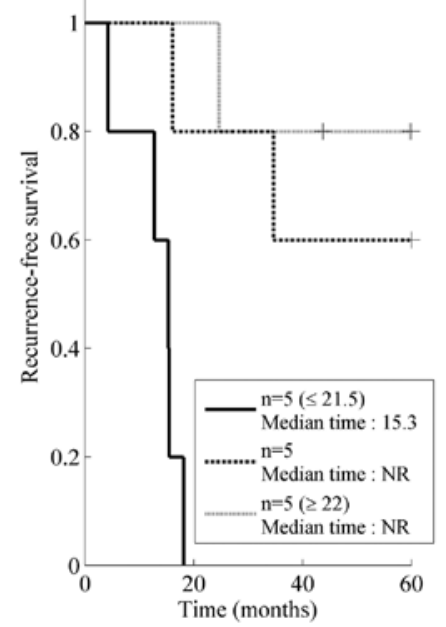

B

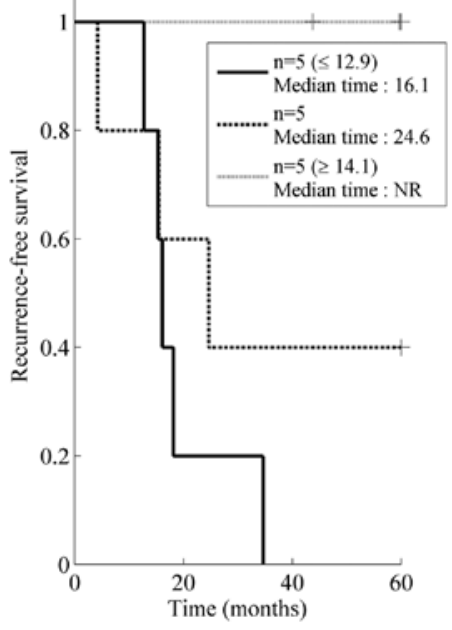

Figure 4. Kaplan-Meier model of recurrence for colon cancer patients using hsa-miR-29a in PCR and microarray platforms. Plots are shown for 15 patients that were measured using PCR (A), with a log-rank p-value of 0.0039 . The plots for these samples as measured in the microarrays is shown as well (B), with a log-rank p-value of 0.004 . Recurrence times after 60 months were censored. NR, not reached. Gray crosses indicate censored patients.

strating, for the first time, the importance of another miRNA, miR-29a, in this setting. Data on miR-29a in CRC is currently limited to two earlier reports. In a study by Huang et al (24), high plasma levels of miR-29a were able to distinguish CRC and advanced adenomas from controls. Moreover, high plasma levels of miR-29a correlated with advanced stage (24). In a later study, by Slattery et al (32), higher expression of miR-29a was found also in CRC samples compared with normal colonic tissues (32).

There are several possible explanations for the discordance between our results and previous ones. One major source of heterogeneity of the findings may be attributable to technical differences in any of the components of the multistep process of the production and evaluation of miRNA. Another possible reason is the different endpoints against which the miRNA expression was tested; whereas some of the studies explored correlations between miRNAs and surrogates for outcome, such as tumor depth of penetration or nodal involvement $(28,30)$. Others including us, evaluated correlations of miRNAs with, the outcome endpoints themselves, DFS, or overall survival (18-21). Furthermore, as all studies reported to date evaluated small cohorts, usually including only a few dozens of patients, the small sample size may also play a major role in the diversity of results. Of note, the inconsistency of data is typical to most studies in this area, with a limited overlap of the findings of the different reports. Taken together, all of this emphasize the preliminary nature of the results which are currently available regarding the prognostic role of miRNA in CRC, including ours. A crucial step forward in that sense would be the performance of large scale validation studies. To date, miR-21 is the only miRNA for which validation data, though limited, was reported (29).

While the actual impact of miR-29a on the clinical course of CRC is still unclear, there are rapidly accumulating clues as to possible mechanisms through which it may influence the cell fate. For example, in vitro analysis of human osteoblasts suggested that Wnt signaling can induce miR-29a transcription, which may then potentiate the Wnt signaling by downregulating key Wnt signaling antagonists. The net result is enhanced Wnt signaling, which is crucial for osteoblast differentiation (33). As Wnt signaling is involved also in colonic cell differentiation (34), the described mechanism may be relevant also to CRC. Other studies have linked miR-29a activity to other proteins/ pathways, including some which play a key role in the biology of CRC, such as c-Myc, NF- $\mathrm{KB}$ and Hedgehog signaling (35), PTEN (36) and p53 (37). Functionally, miR-29a and other members of the miR-29 family were associated with inhibition of cell proliferation, migration and invasion, as well as promotion of apoptosis, in different cancer models (38-40). As one would expect from miRNAs, these effects of the miR29 family were shown to involve interactions with multiple proteins/ pathways (39). If indeed miR-29a is involved with inhibition of the malignant phenotype, regardless of the underlying mechanism, it is not surprising that high expression of this miRNA was associated with a low risk of recurrence in our study.

Several strengths and weaknesses of the current study need to be discussed. First, its cohort, though small, is one of the larger studied in this topic. Second, the study population was strictly defined: all patients were operated in a single medical center, none received adjuvant therapy and all were closely followed, by the same team, for at least three years. Third, the fold change of miR-29a, which is the closest to depict the quantitative difference between the expression levels of this miRNA in the two groups, was relatively small (1.4). Still, this difference reached a highly significant statistical difference $(\mathrm{p}=6.4 \mathrm{e}-5)$. Moreover, a fold change in this order is very common for studies evaluating miRNAs as biomarkers. Fourth, several statistical tests were run to reduce the risk of randomly choosing a 'statistically significant' miRNA from the hundreds tested (multiple hypothesis testing). Fifth, qRT-PCR was used to verify the findings of the miRNA microarrays. Lastly, our results provide clinically meaningful predictive values. We identified a group of patients with high expression of miR-29a for which the PPV for nonrecurrence was $94 \%$. Clearly, if these results are validated, these patients can be safely spared adjuvant chemotherapy.

In summary, this study showed, for the first time, that miR-29a has the potential to serve as a biomarker of the 
risk of recurrence in stage II, but not in stage I colon cancer. Our results add to the accumulating evidence on the critical role of the miR-29 family members in the biology of cancer. Further research in this field is warranted and in this context, a follow-up validation study is planned.

\section{References}

1. Ferlay J, Shin HR, Bray F, Forman D, Mathers C and Parkin DM: Estimates of worldwide burden of cancer in 2008: GLOBOCAN 2008. Int J Cancer 127: 2893-2917, 2010.

2. Green FL: Colon and rectum. In: American Joint Committee on Cancer: AJCC Cancer Staging Manual. 6th edition. LippincottRaven, Philadelphia, PA, 2002

3. Compton CC, Fielding LP, Burgart LJ, et al: Prognostic factors in colorectal cancer. College of American Pathologists Consensus Statement 1999. Arch Pathol Lab Med 124: 979-994, 2000.

4. Kerr D, Gray R, Quirke P, et al: A quantitative multi-gene RT-qPCR assay for prediction of recurrence in stage II colon cancer: Selection of the genes in 4 large studies and results from the independent, prospectively-defined QUASAR calidation study. J Clin Oncol 27: 15s, 2009.

5. Bentwich I, Avniel A, Karov Y, et al: Identification of hundreds of conserved and nonconserved human microRNAs. Nat Gen 37: 766-770, 2005.

6. Farh KK, Grimson A, Jan C, et al: The widespread impact of mammalian microRNAs on mRNA repression and evolution. Science 310: 1817-1821, 2005.

7. Negrini M, Ferracin M, Sabbioni S and Croce CM: microRNAs in human cancer: from research to therapy. J Cell Sci 120 1833-1840, 2007.

8. Calin GA and Croce CM: MicroRNA signatures in human cancers. Nat Rev Cancer 6: 857-866, 2006.

9. Ahmed FE: Role of miRNA in carcinogenesis and biomarker selection: a methodological view. Expert Rev Mol Diagn 7 : 569-603, 2007.

10. Nass D, Rosenwald S, Meiri E, et al: miR-92b and miR-9/9* are specifically expressed in brain primary tumors and can be used to differentiate primary from metastatic brain tumors. Brain Pathol 19: 375-383, 2009.

11. Rosenfeld N, Aharonov R, Meiri E, et al: MicroRNAs accurately identify cancer tissue origin. Nat Biotechnol 26: 462-469, 2008.

12. Brendle A, Lei H, Brandt A, et al: Polymorphisms in predicted microRNA-binding sites in integrin genes and breast cancer: ITGB4 as prognostic marker. Carcinogenesis 29: 1394-1399, 2008.

13. Markou A, Tsaroucha EG,Kaklamanis L, Fotinou M, Georgoulias V and Lianidou ES: Prognostic value of mature microRNA-21 and microRNA-205 overexpression in non-small cell lung cancer by quantitative real-time RT-PCR. Clin Chem 54: 1696-1704, 2008.

14. Hu X, Macdonald DM, Huettner PC, et al: A miR-200 microRNA cluster as prognostic marker in advanced ovarian cancer. Gynecol Oncol 14: 457-464, 2009.

15. Langer C,Marcucci G, Holland KB, et al: Prognostic importance of MN1 transcript levels, and biologic insights from MN1-associated gene and microRNA expression signatures in cytogenetically normal acute myeloid leukemia: a cancer and leukemia group B study. J Clin Oncol 27: 3198-3204, 2009.

16. Wei JS, Johansson $\mathrm{P}, \mathrm{Chen} \mathrm{QR}$, et al: microRNA profiling identifies cancer-specific and prognostic signatures in pediatric malignancies. Clin Cancer Res 15: 5560-5568, 2009.

17. Brenner B, Hoshen M, Purim O, et al: MicroRNAs as a potential prognostic factor in gastric cancer. World J Gastroenterol 17: 3976-3985, 2011.

18. Schetter AJ, Leung SY, Sohn JJ, et al: MicroRNA expression profiles associated with prognosis and therapeutic outcome in colon adenocarcinoma. JAMA 299: 425-436, 2008.

19. Schepeler T, Reinert JT, Ostenfeld MS, et al: Diagnostic and prognostic microRNAs in stage II colon cancer. Cancer Res 68: 6416-6424, 2008
20. Díaz R, Silva J, García JM, et al: Deregulated expression of miR-106a predicts survival in human colon cancer patients. Genes Chromosomes Cancer 47: 794-802, 2008.

21. Xi Y, Formentini A, Chien M, et al: Prognostic values of microRNAs in colorectal cancer. Biomark Insights 2: 113-121, 2006.

22. Gilad S, Meiri E, Yogev Y, et al: Serum microRNAs are promising novel biomarkers. PLoS One 3: e3148, 2008

23. Benjamini Y and Hochberg Y: Controlling the false discovery rate: A practical and powerful approach to multiple testing. J Roy Statist Soc Ser B 57: 289-300, 1995.

24. Huang Z, Huang D, Ni S, Peng Z, Sheng W and Du X: Plasma microRNAs are promising novel biomarkers for early detection of colorectal cancer. Int J Cancer 127: 118-126, 2010.

25. Link A, Balaguer F, Shen Y et al: Fecal microRNAs as novel biomarkers for colon cancer screening. Cancer Epidemiol Biomarkers Prev 19: 1766-1774, 2010.

26. Nakajima G, Hayashi K, Xi Y, et al: Non-coding microRNAs hsa-let-7g and hsa-miR-181b are associated with chemoresponse to $\mathrm{S}-1$ in colon cancer. Cancer Genomics Proteomics 3: 317-324, 2006.

27. Zhang W, Winder T, Ning Y, et al: A let-7 microRNA-binding site polymorphism in 3'-untranslated region of KRAS gene predicts response in wild-type KRAS patients with metastatic colorectal cancer treated with cetuximab monotherapy. Ann Oncol 22: 104-109, 2011.

28. Slaby O, Svoboda M, Fabian P, et al: Altered expression of miR-21, miR-31, miR-143 and miR-145 is related to clinicopathologic features of colorectal cancer. Oncology 72: 397-402, 2007.

29. Kulda V, Pesta M, Topolcan O, et al: Relevance of miR-21 and miR-143 expression in tissue samples of colorectal carcinoma and its liver metastases. Cancer Genet Cytogenet 200: 154-160, 2010.

30. Wang CJ, Zhou ZG, Wang L, et al: Clinicopathological significance of microRNA-31, -143 and -145 expression in colorectal cancer. Dis Markers 26: 27-34, 2009.

31. Motoyama K, Inoue H, Takatsuno Y, et al: Over- and underexpressed microRNAs in human colorectal cancer. Int J Oncol 34: 1069-1075, 2009.

32. Slattery ML, Wolff E, Hoffman MD, Pellatt DF, Milash B and Wolff RK: MicroRNAs and colon and rectal cancer: differential expression by tumor location and subtype. Genes Chromosomes Cancer 50: 196-206, 2011.

33. Kapinas K, Kessler C, Ricks T, Gronowicz G and Delany AM: miR-29 modulates Wnt signaling in human osteoblasts through a positive feedback loop. J Biol Chem 285: 25221-25231, 2010.

34. Fre S, Pallavi SK, Huyghe M, et al: Notch and Wnt signals cooperatively control cell proliferation and tumorigenesis in the intestine. Proc Natl Acad Sci USA 106: 6309-6314, 2009.

35. Mott JL, Kurita S, Cazanave SC, Bronk SF, Werneburg NW and Fernandez-Zapico ME: Transcriptional suppression of mir-29b-1/ mir-29a promoter by c-Myc, hedgehog, and NF-kappaB. J Cell Biochem 110: 1155-1164, 2010.

36. Kong G, Zhang J, Zhang S, Shan C, Ye L and Zhang X: Upregulated microRNA-29a by hepatitis B virus X protein enhances hepatoma cell migration by targeting PTEN in cell culture model. PLoS One 6: e19518, 2011.

37. Park SY, Lee JH, Ha M, Nam JW and Kim VN: miR-29 miRNAs activate $\mathrm{p} 53$ by targeting $\mathrm{p} 85$ alpha and CDC42. Nat Struct Mol Biol 16: 23-29, 2009.

38. Lang N, Liu M, Tang QL, Chen X, Liu Z and Bi F: Effects of microRNA-29 family members on proliferation and invasion of gastric cancer cell lines. Chin J Cancer 29: 603-610, 2010.

39. Muniyappa MK, Dowling P, Henry M, et al: MiRNA-29a regulates the expression of numerous proteins and reduces the invasiveness and proliferation of human carcinoma cell lines. Eur J Cancer 45: 3104-3118, 2009.

40. Han YC, Park CY, Bhagat G, et al: microRNA-29a induces aberrant self-renewal capacity in hematopoietic progenitors, biased myeloid development, and acute myeloid leukemia. J Exp Med 207: 475-489, 2010. 